\title{
Application of Cbct in Periodontics
}

\author{
${ }^{1}$ Melissa L Pereira, ${ }^{2}$ Nagarathna D. V \\ ${ }^{1}$ Postgraduate Student, Department Of Periodontics, A. J Institute Of Dental Sciences, Mangalore, \\ Karnataka, India \\ ${ }^{2}$ Professor, Department Of Periodontics, A. J Institute Of Dental Sciences, Mangalore, Karnataka, India
}

\begin{abstract}
Diagnosis of periodontal disease mainly depends on clinical signs and symptoms. However, in the case of bone destruction, radiographs are valuable diagnostic tools as an adjunct to the clinical examination. Two dimensional periapical and panoramic radiographs are routinely used for diagnosing periodontal bone levels. In two dimensional imaging, evaluation of bone craters, lamina dura and periodontal bone level is limitedby projection geometry and superimpositions of adjacent anatomical structures. These limitations of $2 \mathrm{D}$ radiographs can be eliminated by the use of three-dimensional imaging techniques such as computed tomography. Cone beam computed tomography (CBCT) generates $3 D$ volumetric images. CBCT displays $3 D$ images that are necessary for the diagnosis of intra bony defects, furcation involvements and buccal/lingual bone destructions. CBCT applications provide obvious benefits in periodontics, however; it should be used only considering the necessity and the potential hazards of the examination.
\end{abstract}

Keywords: Cone beam computed tomography, Periodontics, Radiology, Dentistry, Oral diagnosis

\section{Introduction}

Periodontal disease is an inflammatory disease; its diagnosis mainly depends on clinical signs and symptoms. However in case of bone destruction; radiography is a valuable tool as an adjunct to the clinical examination. Although the periapical and panaromic radiographs are routinely used, they may cause magnification and distraction of the image .Conventional radiographs generate 2D images in which the roots are superimposed on the bone and as a result the bone changes such as furcation involvement and buccal and lingual bone changes are difficult to be observed. ${ }^{1}$

Radiographs provide information about the quality localization of the bone defect and the pattern of the bone resorption, changes in the bony trabeculae, condition of the lamina dura, length and shape of the root and the crown root ratio. Some etiological factors like calculus and faulty restoration can also be seen in radiographs. The indications of periapical radiography are the detection of apical infection and inflammation, for periodontal status assessment, for studying the integrity of the lamina dura, for assessing the teeth and the alveolar bone after trauma, for preoperative assessment and postoperative appraisal of apical surgery, for the detailed evaluation of apical cysts and other lesions within the alveolar bone and to evaluate site for implant placement. The Orthopantogram $(\mathrm{OPG})$ is the widely and most commonly used radiographically method for evaluation of alveolar bone levels in periodontal disease, for visualizing the maxilla and mandible in one film, for patient education, for the evaluation of impacted teeth, in the evaluation of multiple unerupted supernumerary teeth, to evaluate traumatic injuries, for radiographic examination of patients with limited mouth opening. In spite of its many valuable uses there are some obvious disadvantages of the OPG such as in the bicuspid area there is overlapping seen in the maxilla and the mandible, the artifacts are easily misinterpreted and can be commonly seen. The most important disadvantage of the intraoral periapical radiography and OPG is that they are $2 \mathrm{D}$ in nature. These disadvantages of the $2 \mathrm{D}$ radiography can be eliminated by using a 3D imaging technique such as the Computed Tomography (CT) ${ }^{3}$

\section{Cone Beam Computed Tomography (Cbct)}

The CBCT scanners work on volumetric tomography. The cone-beam technique involves a single $360^{\circ}$ scan in which there is an $\mathrm{x}$-ray source and a reciprocating area detector which synchronously moves around the patient's head (stabilized with a head holder). At certain degree intervals a single projection image which is known as "basis" image is acquired. This is similar to lateral cephalometric radiographic images, each slightly offset from each another. This series of basis projection images are referred to as the projection data. Software programs incorporating sophisticated algorithms including back-filtered projection are applied to this image data to generate a 3D volumetric data set, which then can be used to provide primary reconstruction images which in axial, sagittal and coronal planes. ${ }^{4}$

The digital imaging has some distinct practical advantages including that in CBCT the collimation of the primary x-ray beam to the area of interest there is a reduction in the size of the irradiated area thereby 
minimizing the radiation dose and it has been seen that CBCT images can result in a low level of metal artifact, particularly in the reconstruction of the jaw and teeth. The resultant images from the CBCT are accurate with submillimeter isotopic voxel resolution ranging from 0.4 to $0.076 \mathrm{~mm}$. When compared to conventional CT, the patient radiation dose is 5 times lower and the exposure time is approximately $1 / 7^{\text {th }}$ the amount of CBCT. ${ }^{4}$

СВCT has been used visualize the interproximal defects,buccal and lingual bone defects ,for diagnosing dehiscence and fenestration defects, for the evaluation of diagnostic and treatment-outcome of periodontitis, postsurgical result evaluation of regenerative periodontal therapy, for the measurement of alveolar bone density for assessing the healing after graft placement in periodontology.

\subsection{CBCT as A Soft tissue Assessment tool}

\section{Diagnostic Importance Of Cbct In Periodontics}

One of the shortcomings of CBCT is that its inability to discriminate soft tissues and that has made CBCT is a more appropriate tool for the evaluation of mineralized tissue.So, a method called Soft tissue CBCT (ST CBCT) was introduced and was used to determine the dimension and the relationship of the structure of dentogingival unit. In this method, during the CBCT scanning the patients tongues were retracted toward the floor of their mouths and a plastic lip retractor was used to retract the soft tissues away from the teeth and gingiva and the resultant images gave clear information for the analysis of various dentogingival unit measurements. ${ }^{10}$ In a another study where ST CBCT method was usedin which the average thickness of the palatal mucosa according to age and specific localizations was determined, the authors concluded that the method could be used to consistently obtain high-quality images of the palatal masticatory mucosa. ${ }^{11}$ As the thickness of palatal mucosa is important for the treatment planning of soft tissue grafts, and this technique which is used only provides quantitative assessment not qualitative assessment, the differences between the epithelial, fat and connective tissues cannot be distinguished on the Soft tissue $\mathrm{CBCT}^{10}$

\subsection{Periodontal Ligament Space}

The earliest sign of periodontitis that can be detected on a radiograph is by viewing the continuity of the lamina dura. Whenever the continuity is lost, some changes where observed in the periodontal ligament space. Ozmeric et al. in his study in order to compare between CBCT and conventional radiographs created a phantom model with artificial periodontal ligament space and had found that the Periapical radiographs were superior to CBCT for the measurement of periodontal ligament space ${ }^{12}$. But conflicting results were reported by the authors of another in vitro study that found CBCT to be better than conventional radiography in visualizing the periodontal ligament $\operatorname{space}^{13}$.Still, anaccurate method of assessing periodontal ligament space requires some further research.

\subsection{Alveolar Bone Defect}

Radiograph should be precise in order to assess the bony defect. Two Dimensional radiographs can be insufficient for the detection of intrabony alveolar defects due to the obstruction of spongious bone changes by cortical plate. In a study where the authors had compared the image quality between CBCT and conventional radiography they had concluded with the result that the CBCT scanner provided better diagnostic and quantitative information on periodontal bone levels in three dimensions than conventional radiography. However, it was seen thatin both the techniques the accuracy in the anterior aspect of the jaws was limited. ${ }^{14}$ In a study done by B Vandenberghe et al where he had evaluated the Detection of periodontal bone loss using digital intraoral and cone beam computed tomography image he had found that there was no significant difference the two methods ${ }^{5}$. In Misch and colleagues study they demonstrated that CBCT was as accurate as direct measurements using a periodontal probe and as reliable as radiographs for interproximal areas. ${ }^{15}$ In their 2005 study, Mengel and coworkersinvestigated the use of CBCT in the diagnosis of periodontal defects It was demonstrated that all intrabony defects could be measured in three planes in the CBCT scans with great accuracy true to scale. ${ }^{16}$ Similar results were found by Noujeimand and coworkers when using the CBCT system to detect simulated interradicular lesions of varying depth in comparison with intraoral radiography. ${ }^{17}$ Rees et al. had found that proximal osseous defects and furcation defects on the facial and lingual surfaces of multi-rooted teeth could be identified with a high degree of accuracy by their radiographic appearances using CBCT. Conversely, lesions on the facial or lingual root surfaces are extremely difficult to recognize radiographically. They had also found it difficult to diagnose the furcation involvement of mandibular left first molar on IOPA. ${ }^{18}$

\section{CBCT in Regenerative Therapy 4.1 Furcation Involvement}

Radicular bone assessment is a necessary step in furcation involvement treatment planning.Conventional radiographs can be deceptive in the evaluation of the bone support in the intraradicular 
area particularly the maxillary molar.Detailed information of furcation involvement was given by the CBCT images of maxillary molars ${ }^{7}$. Studies reported that intra-surgical findings confirmed with $82.4 \%$ of the CBCT data when CBCT's accuracy was accessed in the maxillary molar and it was seen that the horizontal bone loss and vertical bone loss were under-estimated by $\mathrm{CBCT}^{8}$. When $\mathrm{CBCT}$ images of artificially created furcation involvement of the second molars in pig mandibles was evaluated it was seen that it had an accuracy ranging from $78 \%$ to $88 \%$ leading to the author to conclude that CBCT can be considered as a reliable and accurate method for detecting incipient furcation involvement. ${ }^{9}$ In another study the author where the author had compared CBCT to intraoral radiography he had concluded that the detection of crater and furcation involvements had failed in $29 \%$ and $44 \%$ for the intraoral radiograph, respectively, as compared to $100 \%$ detectability for both defects with CBCT. ${ }^{5}$

\subsection{Regenerative Periodontal therapy and Bone Graft}

In their study, Grimard et al compared direct clinical, periapical radiograph, and CBCT measurement techniques for assessing bone level changes following regenerative periodontal therapy in 35 intrabony defects. It was found out that CBCT was significantly more precise and accurate than periapical radiographs and concluded that CBCT may obviate surgical reentry as a technique for assessing regenerative therapy outcomes. ${ }^{18}$ Ito et al, used CBCT to evaluate the outcome of regenerative therapy. The CBCT images allowed observation of the morphology of periodontal bone defects in three dimensions; only the axial dimension was used to make a template for the GTR membrane.By using the template for the membrane it was seen that the membrane could be easily trimmed and adapted to the root surface on either the maxillary or mandibular arch. On the other hand,without the use of CBCT, it was seen that the membrane had a larger interproximal area,and the mesial buccal site was smaller. The membrane trimming time was seen to be shortened when the CBCT was used. ${ }^{20} \mathrm{CBCT}$ was also seen to give an accurate measurement in the calculation of the volume of alveolar defect and bone graft in alveolar cleft patients. Preoperative scans could aid in quantitatively determining the bone amount needed to adequately fill the bone defect, and the postoperative scans give accurate follow-up evaluation after surgery. ${ }^{21}$

\section{Cbct In Implant Planning}

Radiographs play a very important role in the assessment of bone in Implant planning. CBCT increases the accuracy of treatment planning in predicting implant length defined at surgery. In relation to the different regions, i.e., maxilla and mandible (posterior or anterior), Hu etal.evaluated not only the reliability of the two presurgical preparation methods, PAN radiography and CBCT, but also the surgery stage. The result shows that implant planning can be performed safely using digital PAN radiography for the mandible, but CBCT was recommended for the maxilla ${ }^{22}$.Correa et al.found that implant sizes measured using CS CBCT images were both narrower and shorter than the sizes obtained from digital PAN radiographs and CBCT-PAN views; the difference in width selection could be observed in the upper premolar region, and the difference in length, in the lower molar region ${ }^{23}$. In the study done by Ekrish, to investigate the effect of exposure time on the accuracy and reliability of cone beam computed tomography in the assessment of dental implant site dimensions in dry skulls which showed that lowering the CBCT exposure time does not affect the reliability or accuracy of implant site measurements. ${ }^{24}$ The use of CBCT in implant dentistry has reduced implant failures by providing information about bone density, the shape of the alveolus, and the height and width of the proposed implant site for each patient $^{25}$ and CBCT's effectiveness in quantifying and assessing the shape of the alveolus has led to improved case selection. CBCT is commonly utilized in postsurgical evaluations to assess bone grafts and the implant's position in the alveolus 26

\section{Conclusion}

In Periodontology, CBCT has the potential to gather accurate diagnostic and quantitative information about bone condition. Bony defects, caters, and furcationinvolvements seem to be better depicted on CBCT, whereasbone quality and periodontal ligament space scored betteron conventional intraoral radiography. CBCT does not offera significant advantage over conventional radiography forassessing the periodontal bone levels. In the field of Implantology also CBCT has been seen to be effective in providing essential information and the bone morphology and for implant site measurements. The decision pertaining tothe use of CBCT in the field of periodontology should betaken after careful consideration of its advantages, limitations, and risks.

\section{References}

[1]. Marjorie K. Jeffcoat, I.-Chung Wang,Michael S. Reddy; Radiographic diagnosis in periodontics, Perio 2000;1995; 7 (1):54-68

[2]. EF Corbet, DKL Ho, SML Lai Radiographs in periodontal disease diagnosis andmanagement.Australian Dental Journal2009; 54:(1 Suppl): S27-S43

[3]. White SC, PharoahMJ.Oralradiology:principles and interpretation (United States,Elsevier Health Sciences;2014)

[4]. Clinical Applications of Cone-Beam Computed Tomography in Dental Practice; J Can Dent Assoc 2006; 72(1):75-80 
[5]. Detection of periodontal bone loss using digital intraoral and cone beam computed tomography images: an in vitro assessment of bony and/or infrabony defects B Vandenberghe R Jacobs and J Yang Dentomaxillofacial Radiology (2008) 37, 252-260

[6]. Shah MA, Shah SS, Dave DH. 'CBCT-A positive amelioration in periodontics'.NJIRM 2013; 4(3) : 144-148

[7]. Walter C, Kaner D, Berndt DC, Weiger R, ZitzmannNU.Three-dimensional imaging as a pre-operative tool in decisionmaking for furcation surgery. J ClinPeriodontol2009; 36: 250-257

[8]. Qiao J, Wang S, Duan J, Zhang Y, Qiu Y, Sun C, Liu D. The accuracy of conebeamcomputed tomography in assessing maxillary molar furcation involvement. JClinPeriodontol 2014; 41: 269-274

[9]. Umetsubo OS, Gaia BF, Costa FF, Cavalcanti MG. Detection of simulated incipient furcation involvement by CBCT: an in vitro study using pig mandibles. Brazilian oral research. 2012 Aug;26(4):341-7.

[10]. Januário AL, Barriviera M, Duarte WR. Soft tissue conebeam computed tomography: a novel method for the measurement of gingival tissue and the dimensions of the dentogingival unit. J EsthetRestor Dent 2008; 20: 366-373

[11]. Barriviera M, Duarte WR, Januário AL, Faber J, Bezerra AC. A new method to assess and measure palatal masticatory mucosa by cone-beam computerized tomography. J ClinPeriodontol2009; 36: 564

[12]. Özmeric N, Kostioutchenko I, Hägler G, Frentzen M, Jervøe-Storm PM. Cone-beam computed tomography in assessment of periodontal ligament space: in vitro study on artificial tooth model. Clinical oral investigations. 2008 Sep 1;12(3):233-9.

[13]. Jervøe-Storm PM, Hagner M, Neugebauer J, Ritter L, Zöller JE, Jepsen S, Frentzen M. Comparison of cone-beam computerized tomography and intraoral radiographs for determination of the periodontal ligament in a variable phantom. Oral Surg Oral Med Oral Pathol Oral RadiolEndod2010; 109: e95-e101

[14]. Mol A, Balasundaram A. In vitro cone beam computed tomography imaging of periodontal bone. Dentomaxillofacial Radiology 2014;37(6): 319-324.

[15]. Misch KA, Yi ES, Sarment DP. Accuracy of cone beam computed tomography for periodontal defect measurements. J Periodontol 2006;77: 1261-6.

[16]. Mengel R, Candir M, Shiratori K, et al. Digital volume tomography in the diagnosis of periodontal defects: an in vitro study on native pig and human mandibles. J Periodontol 2005;76(5):665-73.

[17]. Noujeim M, Nummikoski P, Langlais R. Evaluation of high-resolution cone-beam computed tomography in the detection of simulated interradicular bone lesions. Oral Surg Oral Med Oral Pathol Oral RadiolEndod 2007;103: e52.

[18]. Grimard BA, Hoidal MJ, Mills MP, Mellonig JT, Nummikoski PV, Mealey BL. Comparison of clinical, periapical radiograph, and cone-beam volume tomography measurement techniques for assessing bone level changes following regenerative periodontal therapy. J Periodontol. 2009;80:48-55.

[19]. Rees T, Biggs NL, Collins CK. Radiographic interpretation of periodontal osseous defects. Oral Surg Oral Med Oral Pathol Oral RadiolEndod. 1971;2:141-53.

[20]. Takane M, Sato S, Suzuki K, Fukuda T, Asano Y, Honda K, Arai Y, Ito K. Clinical application of cone beam computed tomography for ideal absorbable membrane placement in interproximal bone defects. Journal of oral science. 2010;52(1):63-9.

[21]. Zhou WN, Xu YB, Jiang HB, Wan L, Du YF. Accurate Evaluation of Cone-Beam Computed Tomography to Volumetrically Assess Bone Grafting in Alveolar Cleft Patients.JCraniofac Surg. 2015;26(6):e535-9.

[22]. Hu KS, Choi DY, Lee WJ, Kim HJ, Jung UW, Kim S. Reliability of two different presurgical preparation methods for implant dentistry based on panoramic radiography and cone-beam computed tomography in cadavers. J Periodontal Implant Sci. 2012; 42(2):39-44.

[23]. Correa LR, Spin-Neto R, Stavropoulos A, Schropp L, da Silveira HE, Wenzel A. Planning of dental implant size with digital panoramic radiographs, CBCT-generated panoramic images, and CBCT cross-sectional images.Clin Oral Implants Res. 2014;25(6):690-5.

[24]. Al-Ekrish AA. Effect of exposure time on the accuracy and reliability of cone beam computed tomography in the assessment of dental implant site dimensions in dry skulls. The Saudi Dental Journal. 2012;24(3):127-34.

[25]. Hua Y, Nackaerts O, Duyck J, Maes F, Jacobs R.Bone quality assessment based on cone beam computed tomography imaging. Clin Oral Implants Res 2009;20(8):767-771.

[26]. Tischler M. In-office cone beam computerized tomography: Technology review and clinical examples. Dent Today 2008;27(6):102106. 